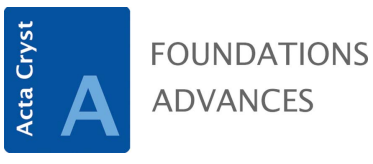

ISSN 2053-2733

Received 28 October 2015

Accepted 14 December 2015

Edited by J. Miao, University of California, Los Angeles, USA

Keywords: coherent X-ray diffraction imaging; $\mathrm{X}$-ray free-electron lasers; frozen-hydrated noncrystalline samples; structural analysis; correlative microscopy.

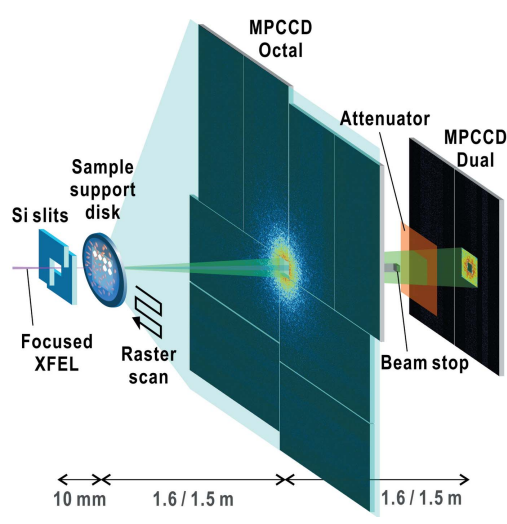

C 2016 International Union of Crystallography

\section{Cryogenic coherent X-ray diffraction imaging of biological samples at SACLA: a correlative approach with cryo-electron and light microscopy}

\author{
Yuki Takayama* and Koji Yonekura*
}

Biostructural Mechanism Laboratory, RIKEN SPring-8 Center, 1-1-1 Kouto, Sayo, Hyogo 679-5148, Japan. *Correspondence e-mail: takayama@spring8.or.jp, yone@spring8.or.jp

Coherent X-ray diffraction imaging at cryogenic temperature (cryo-CXDI) allows the analysis of internal structures of unstained, non-crystalline, whole biological samples in micrometre to sub-micrometre dimensions. Targets include cells and cell organelles. This approach involves preparing frozen-hydrated samples under controlled humidity, transferring the samples to a cryo-stage inside a vacuum chamber of a diffractometer, and then exposing the samples to coherent X-rays. Since 2012, cryo-coherent diffraction imaging (CDI) experiments have been carried out with the X-ray free-electron laser (XFEL) at the SPring-8 Ångstrom Compact free-electron LAser (SACLA) facility in Japan. Complementary use of cryo-electron microscopy and/or light microscopy is highly beneficial for both pre-checking samples and studying the integrity or nature of the sample. This article reports the authors' experience in cryo-XFELCDI of biological cells and organelles at SACLA, and describes an attempt towards reliable and higher-resolution reconstructions, including signal enhancement with strong scatterers and Patterson-search phasing.

\section{Introduction}

Since the first demonstration of coherent X-ray diffraction imaging (CXDI) (Miao et al., 1999), its potential has been actively studied in both material and biological sciences (Miao et al., 2015). In CXDI, spatially coherent X-rays irradiate an isolated sample object and yield a Fraunhofer diffraction pattern of the object on an Ewald sphere (Fig. 1). The pattern must be recorded on an area detector under the oversampling (OS) condition (Miao et al., 2003), which enables recovery of phase information directly from the diffraction intensity by using an iterative phase retrieval (PR) algorithm (Fienup, 1982). Thus, we can obtain a projected electron-density map of a sample object within a given spatial resolution, where the curvature of the Ewald sphere is regarded as a flat plane perpendicular to the incident X-ray beam (projection approximation; Chapman et al., 2006). The long penetration depth and short wavelength of hard X-rays allow visualization of internal structures of whole objects, which are too thick for electron microscopy (EM), and beyond the resolution limit of light microscopy (LM).

Recently, X-ray free-electron laser (XFEL) sources (Emma et al., 2010; Ishikawa et al., 2012) have provided new opportunities in CXDI. Intense, hard X-ray pulses with nearly $100 \%$ spatial coherence and $\sim 10$ fs duration enable diffraction data collection before radiation damage occurs, though samples irradiated with an XFEL pulse are destroyed at an atomic level (Neutze et al., 2000). This advantage is clearly demonstrated in a study of damage-free structures up to at least $\sim 2 \AA$ resolution by XFEL protein crystallography (Hirata et 
al., 2014). XFEL-coherent diffraction imaging (CDI) has so far revealed 30-100 nm resolution structures of non-crystalline biological samples such as viruses (Seibert et al., 2011; Kassemeyer et al., 2012), cellular components (Hantke et al., 2014; Takayama, Inui et al., 2015; Oroguchi et al., 2015) and bacterial cells (Kimura et al., 2014; van der Schot et al., 2015; Oroguchi et al., 2015). In addition, the high repetition rate of XFEL pulses yields much diffraction data in a short time, allowing reconstruction of three-dimensional structures of homogeneous samples (Ekeberg et al., 2015) and statistical analysis of both the shape and internal structures of inhomogeneous samples (Takahashi et al., 2013).

For biological samples, it is critical to maintain their hydrated state throughout the experiment. Imaging before sample destruction with XFEL enables one to study cells in solution (Kimura et al., 2014) or before drying (van der Schot et al., 2015). Living cell morphologies, however, may change during the experiment or isolated cell organelles are often unstable and deteriorate. Cryo-XFEL-CDI can help solve this (Takayama \& Nakasako, 2012; Nakasako et al., 2013; Takayama, Inui et al., 2015; Oroguchi et al., 2015). Flashcooling of samples can retain hydrated states and the integrity of cellular and sub-cellular structures (McDowall et al., 1983) even in a vacuum. Frozen samples can be stored in liquid nitrogen and large amounts of intact samples be supplied for the best use of the XFEL beam. This potentially allows study of cells at a particular period of the cellular cycle. Furthermore, signal-to-noise $(\mathrm{S} / \mathrm{N})$ ratios of diffraction data can be improved by reducing surrounding ice.

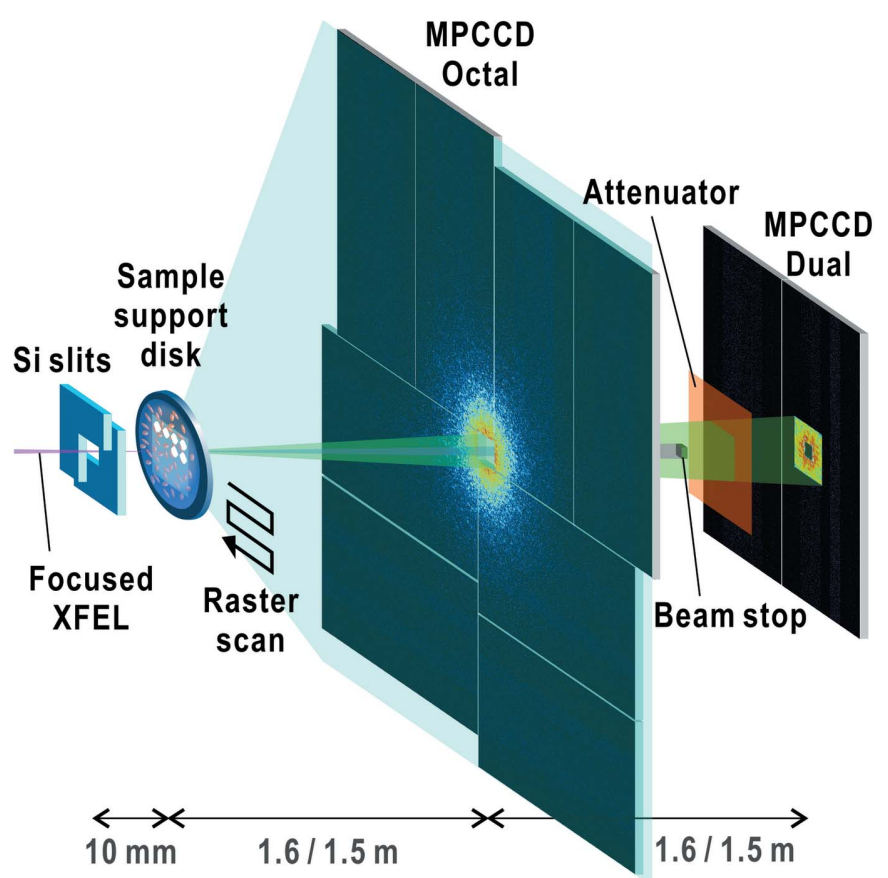

Figure 1

Schematic illustration of the cryo-XFEL-CDI experiment. The distances of the sample to the MPCCD Octal detector and the Octal to Dual detectors are both $\sim 1.6 \mathrm{~m}$ for the KOTOBUKI-1 diffractometer and $\sim 1.5 \mathrm{~m}$ for the TAKASAGO-6.
We have conducted a series of cryogenic XFEL-CDI experiments at the SPring-8 Ångstrom Compact free-electron LAser (SACLA) facility in Japan since 2012. This article reports on our progress and touches on the current status of cryo-XFEL-CDI of biological samples. We discuss future applications in higher spatial resolution analysis and robust phasing. We also show the benefits of complementary use of cryo-EM and LM to answer biological problems.

\section{Methods}

2.1. Preparation of frozen-hydrated samples and pre-check with cryo-EM and LM

We modified the sample preparation protocol widely adopted in cryo-EM (Fig. 2a; Takayama \& Nakasako, 2012; Takayama, Inui et al., 2015). For sample support, we prepared a 20 nm-thick carbon membrane adhered onto a custommade metal disc with multiple measurement windows (Nakasako et al., 2013) or a $100 \mathrm{~nm}$-thick silicon nitride membrane (Norcada, Canada) with carbon deposition. The membrane surface was treated with $0.1 \%(w / v)$ polylysine solution (Sigma-Aldrich, USA), which increases the affinity of the surface for biological samples (Takayama, Inui et al., 2015). Then, we used a custom-made humidity-controlling device with a light microscope (Fig. 2b; Takayama \& Nakasako, 2012). Approximately $2 \mu$ l of concentrated sample suspension was directly applied on the support membrane under controlled relative humidity $(\mathrm{RH})$ of $\sim 100 \%$. After a few minutes, excess solution was blotted off while monitoring the amount of solution remaining, the number density of sample particles etc. with the scope. The sample support disc was transferred to a freezing device while keeping RH higher than $90 \%$ and rapidly frozen by plunging it into liquid ethane.

The frozen samples were examined with cryo-EM and/or cryo-LM. We used a JEM-2100 electron microscope (JEOL, Japan) operated at an accelerating voltage of $200 \mathrm{kV}$ with a 626 cryo-sample holder (Gatan Inc., USA) and a BX51 light microscope (Olympus, Japan) with a CLM77K cryo-sample stage (Instec Inc., USA). If the number density of target objects and the ice thickness appeared suitable, several more samples were frozen in the same way. The humidity-controlling device made the procedure highly reproducible. The samples were stored in liquid nitrogen until cryo-XFEL-CDI experiments. When necessary the samples examined by cryoEM and cryo-LM can also be recovered just by keeping them cold. The devices used for sample preparation are installed in a biological sample room next to the experimental hall of SACLA. Hence, experimental results can be quickly used to feedback to the next sample preparation even during a period of limited beamtime.

\subsection{Cryo-XFEL-CDI experiments at SACLA}

A series of cryo-XFEL-CDI experiments have been performed at SACLA BL3 (Tono et al., 2013) since March 2012. The experimental setup is illustrated in Fig. 1. We used KOTOBUKI-1 diffractometers (Nakasako et al., 2013; 
(a)

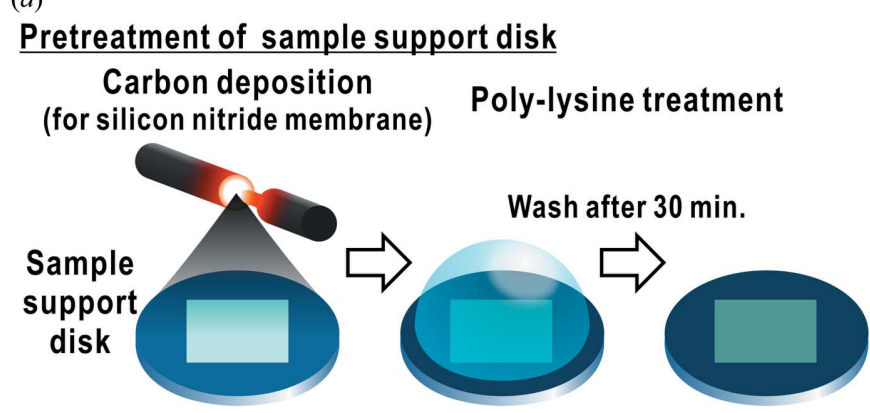

Sample preparation under high $\mathrm{RH}$

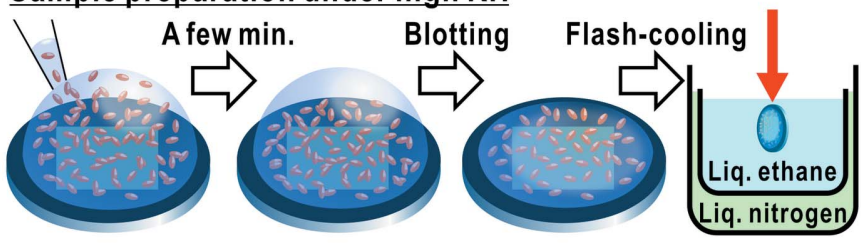

(b)

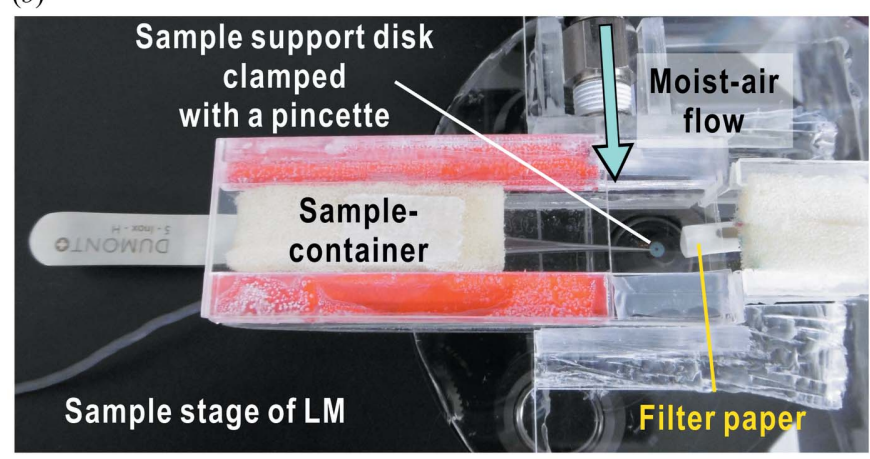

(c)

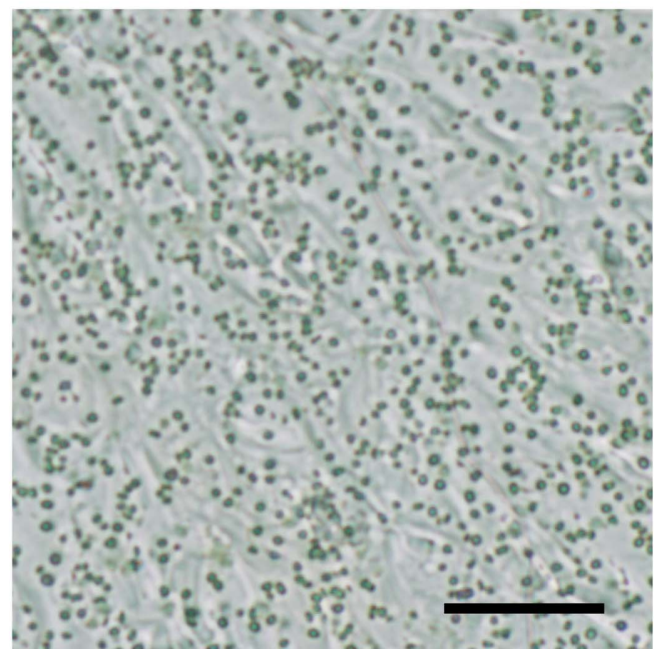

Figure 2

Preparation of frozen-hydrated biological samples. (a) Procedures for pre-treatment of the sample support disc and preparation of frozenhydrated biological samples under controlled RH. (b) The sample preparation chamber attached to the sample stage of the light microscope. The sample container can be detached from the humidity chamber and attached to a freezing device while maintaining humidity (Takayama \& Nakasako, 2012). (c) LM observation of a frozen-hydrated sample of chloroplasts isolated from $C$. merolae, taken at a sample temperature of $82 \mathrm{~K}$. The sample was frozen on a silicon nitride membrane under $\sim 100 \% \mathrm{RH}$ as in $\S 2$ and panel $(a)$. The bar represents $50 \mu \mathrm{m}$.
Oroguchi et al., 2015) until July 2014 and TAKASAGO-6 from March 2015. Inside the vacuum chamber of both diffractometers, a cryogenic pot is placed on a motorized translation stage. The pot is filled with liquid nitrogen and cooled to $\sim 66 \mathrm{~K}$ with evaporative cooling. A frozen sample support disc transferred into the pot can be kept at $\sim 66 \mathrm{~K}$ through thermal contact during X-ray exposures.

Either diffractometer was installed downstream of Kirkpatrick-Baez focusing mirror optics (Yumoto et al., 2012), with the sample position set at the focal spot of the mirrors. The mirrors focus the X-ray beam to $\sim 1.5 \times 1.5 \mu \mathrm{m}$ at halfmaximum intensity $(\sim 2.0 \times 2.0 \mu \mathrm{m}$ at half-maximum amplitude) and a single pulse of $5.5 \mathrm{keV} X$-ray delivers $\sim 10^{10}-10^{11}$ photons to the focal spot (Oroguchi et al., 2015). A pair of L-shaped Si slits placed $10 \mathrm{~mm}$ upstream of the sample position removes almost all parasite scattering from the beamline optics to a resolution of 500-600 nm (Oroguchi et al., 2015). Multi-port CCD (MPCCD) Octal and Dual detectors (Kameshima et al., 2014) disposed in tandem (Fig. 1) record the diffraction pattern with a resolution range of approximately 7-200 $\mathrm{nm}$ and $80-550 \mathrm{~nm}$, respectively. A beam stop of $2 \times 2 \mathrm{~mm}$ and aluminium attenuators with five variable thicknesses $(15-100 \mu \mathrm{m})$ are inserted in front of the Dual detector. To obtain diffraction patterns with optimal S/N ratios, we adjusted the central opening of the Octal detector from 9 to $3.5 \mathrm{~mm}$ and changed the attenuator to avoid pixel saturation.

An XFEL pulse damages samples around the irradiation center. The damaged area extends to at least $15 \mu \mathrm{m}$ (Nakasako et al., 2013; Hirata et al., 2014). Hence, we raster-scanned the sample support disc with a step of $25-50 \mu \mathrm{m}$ to provide a fresh area for every exposure. The scheme for the scan can be edited in a GUI window of the IDATEN control-software suite, and is then submitted to a controller of the sample stage to synchronize movement of the sample stage and exposure of XFEL pulses (Sekiguchi, Yamamoto et al., 2014).

\subsection{Data processing}

After each raster scan, diffraction data sets were processed by the G-SITENNO suite (Sekiguchi, Oroguchi et al., 2014; Sekiguchi, Yamamoto et al., 2014) installed on an HPC cluster system at SACLA (Joti et al., 2015). The software suite automatically subtracts the dark current of the detector from diffraction patterns, merges pairs of the patterns recorded on the Octal and Dual detectors, and identifies patterns with a given level of signals as 'hit' from a huge number of patterns (Sekiguchi, Oroguchi et al., 2014). Then it calculates preliminary image reconstruction from all the hit patterns by using hybrid input-output (Fienup, 1982) and shrink-wrap (Marchesini et al., 2003) (HIO-SW) algorithms.

G-SITENNO also provides statistics of diffraction data sets and a graphical summary of the preliminary reconstruction (Sekiguchi, Yamamoto et al., 2014). Based on the information, we can adjust conditions for the next diffraction measurement and/or sample preparation during a single beamtime session. 
Table 1

Statistics of diffraction patterns and PR calculations.

Correlation of the Friedel mate on the diffraction pattern is calculated as follows: $C_{\text {sym }}=(E-O) /(E+O), E=\sum_{x, y}\left[I_{0}(x, y)+I_{\text {sym }}(-x,-y)\right]^{2}$, $O=\sum_{x, y}\left[I_{0}(x, y)-I_{\text {sym }}(-x,-y)\right]^{2}$ where $I_{0}(x, y)$ and $I_{\text {sym }}(-x,-y)$ represent the diffraction intensity in the region of interest with $100 \times 100$ pixels and its Friedel mate, respectively. When the diffraction pattern displays the ideal Friedel symmetry, the $C_{\text {sym }}$ value becomes 1 (Sekiguchi, Oroguchi et al., 2014).

Maximum resolution is defined as the highest resolution shell including at least two detector pixels with four photons or more (Sekiguchi, Yamamoto et al., 2014).

The OS ratio of the reconstructed map is defined as the number of pixels in the diffraction patterns over the number of pixels in a support of the map, which corresponds to the redundancy of the data (Miao et al., 2003).

$R_{\mathrm{F}}$ is defined as $R_{\mathrm{F}}=\sum|| F_{\text {obs }}|-K| F_{\text {calc }}|| / \sum\left|F_{\text {obs }}\right|$, where $\left|F_{\text {obs }}\right|$ and $\left|F_{\text {calc }}\right|$ are the observed structural amplitude and that calculated from the averaged electrondensity map. $K$ is a scale factor between both the amplitudes (Miao et al., 2006).

The effective resolution of the averaged electron-density map is defined as the resolution where the PRTF drops below 0.5 (Chapman et al., 2006). The calculation only includes pixels with four photons or more.

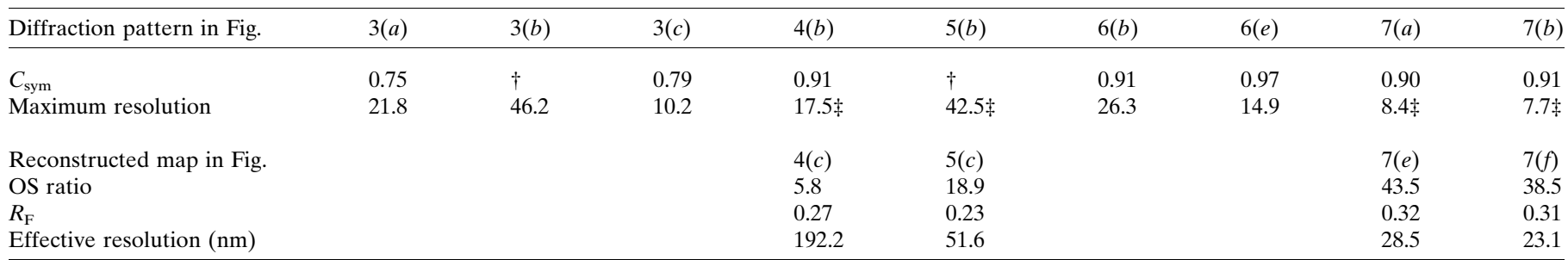

$\dagger$ We were not able to assign enough area to calculate the $C_{\text {sym }}$ value for the diffraction patterns in Figs. $3(b)$ and $5(b)$ as the patterns show low diffraction signals. $\ddagger$ Maximum resolution of the diffraction patterns for the structural analysis was calculated after $2 \times 2$ binning (see $\$ 2$ ).

\subsection{Structural analysis}

Reconstruction of projected electron-density maps from diffraction patterns was carried out on the HPC systems at SACLA. To improve $\mathrm{S} / \mathrm{N}$ ratios, diffraction patterns were first $2 \times 2$ binned, which kept the two-dimensional OS ratios $\sim 6$ or higher (Table 1). The data binning does not affect the quality of reconstructions when the OS ratio is higher than 5 (Miao et al., 2003). The PR calculation needs to define the 'support', which should roughly enclose target objects. As an initial estimate of the support, we usually used a loose envelope of autocorrelation of the sample (Marchesini et al., 2003) obtained by calculating the Patterson map, which is a Fourier transform of the diffraction pattern. The initial support was also derived from cryo-EM observation for a chloroplast or by the Patterson-search phasing method (Takayama, MakiYonekura et al., 2015) for samples containing colloidal gold particles. Then, starting from random densities and the support, we carried out hundreds to thousands of independent HIO-SW PR calculations to produce initial reconstructions of the target objects. A principal component analysis (PCA; Sekiguchi et al., 2016) was applied to select the most probable group of the initial reconstructions. The top 100 reconstructions that best represented the group were averaged and used to refine the support. When starting from the support determined by Patterson-search phasing, the PR calculations converged to a well defined solution, and this case did not need PCA as previously reported (Takayama, Maki-Yonekura et al., 2015).

We again repeated hundreds to thousands of independent PR calculations with the refined support and random densities by using the oversampling smoothness (OSS) algorithm (Rodriguez et al., 2013). We adopted the final projected electron-density map by averaging 100 of the most reliable reconstructions through PCA. The effective resolution of the projection map was taken to be the resolution where the phase retrieval transfer function (PRTF; Chapman et al., 2006) of the map drops below 0.5 .

\section{Results and discussion}

\subsection{Sample preparation for efficient collection of optimal} diffraction signals from biological samples

The data collection efficiency per sample support disc strongly depends on the number density of target objects, yet isolated objects are preferable for the PR calculation. Thus, it is ideal to have a high number density of mono-dispersed particles on the support membrane. Biological samples, however, tend to aggregate when blotting off excess solution due to a low affinity between membrane and sample. We found that a carbon membrane with polylysine treatment was very effective in yielding suitable samples (Takayama, Inui et $a l ., 2015)$. By adjusting the concentration of chloroplasts from red algae Cyanidioschyzon merolae, we prepared frozenhydrated samples as in Fig. 2(c). Here there are approximately 2-5 chloroplasts per $(10 \times 10 \mu \mathrm{m})$, which is a suitable concentration for cryo-XFEL-CDI. This number density is only reproducible with membranes treated with polylysine.

Diffraction patterns obtained by cryo-XFEL-CDI are strongly affected by several factors such as the incident-beam intensity, the relative position of sample objects to the beam center and the total scattering cross section of samples within the beam. In addition, the humidity during sample preparation and the thickness of surrounding ice are also critical to obtain optimal diffraction signals from biological samples (Fig. 3). Chloroplast samples prepared under $100 \%$ RH and embedded in thin vitreous ice gave good diffraction signals (Fig. 3a; see also Table 1 for the statistics). The pattern is composed of 
speckles with $\sim 5 \times 5$ pixels, which relates to a single chloroplast with a dimension of $\sim 1.5 \mu \mathrm{m}$. The characteristic features in the pattern probably reflect internal structures of the chloroplast. In contrast, diffraction patterns obtained from samples prepared below $90 \%$ RH show much weaker signals (Fig. $3 b$ and Table 1), suggesting that finer chloroplast structures were destroyed at the lower humidity. Samples embedded in thicker ice also yielded strong diffraction patterns extending over wider diffraction angles (Fig. $3 c$ and Table 1). However the pattern shows fewer features and much smaller speckles, likely reflecting an ice blob. The signals from the biological objects are of little use here as they are weaker than those from the thick ice.

As demonstrated above, cryo-XFEL-CDI of biological samples is highly reliant on suitable samples. Hence a precheck of samples with cryo-EM and/or LM is very helpful for
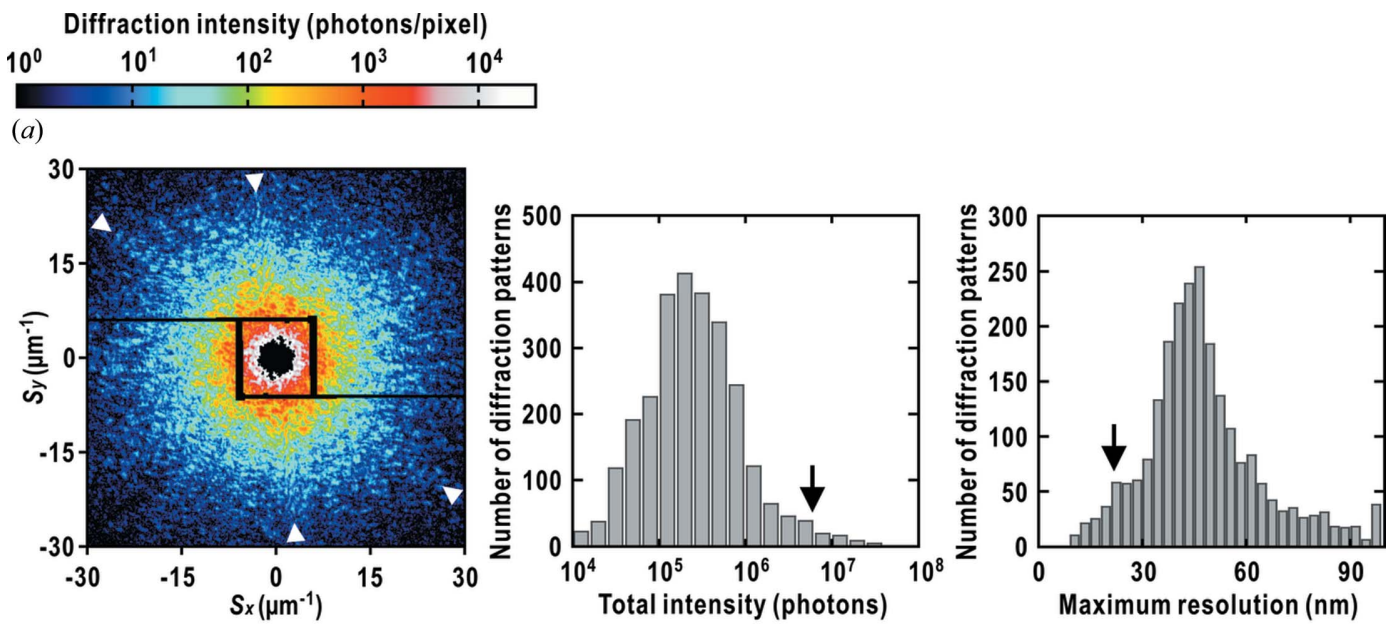

(b)
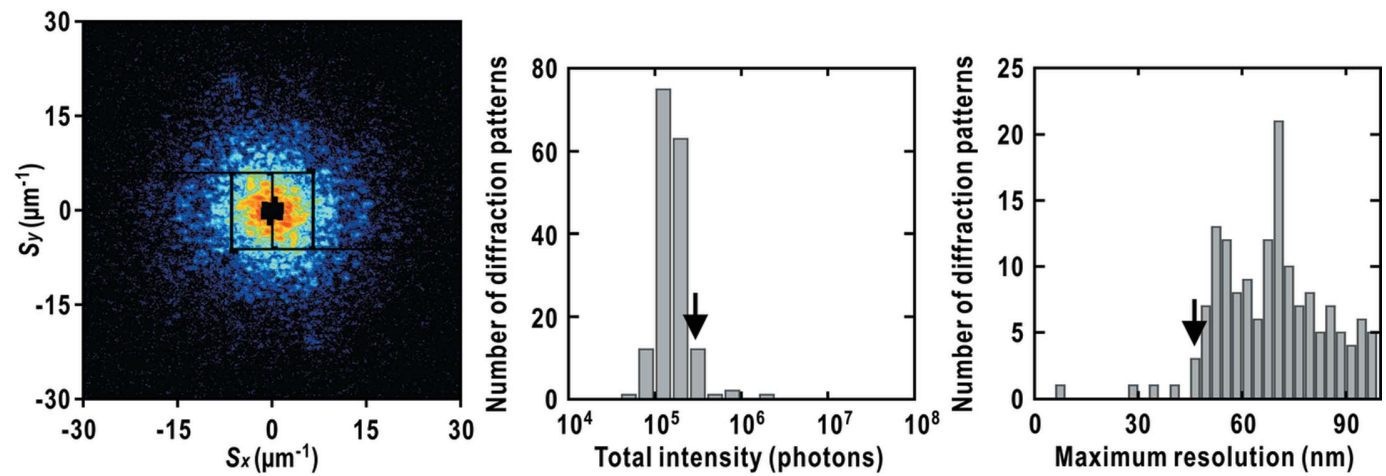

(c)
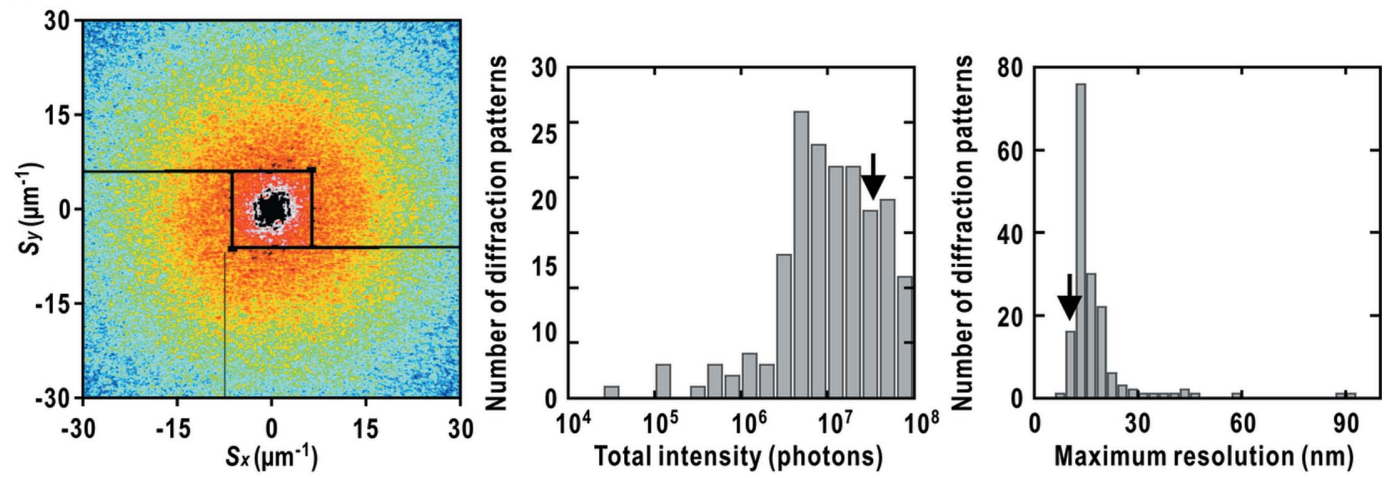

Figure 3

Diffraction patterns from frozen-hydrated chloroplast samples. Diffraction data were collected from chloroplasts frozen in thin vitreous ice under $\sim 100 \% \mathrm{RH}(a)$, below $90 \% \mathrm{RH}(b)$, embedded in thicker ice $(c)$. Figures from the left to right columns correspond to representative diffraction patterns showing higher signals, histograms of total intensity over diffraction data sets collected from samples frozen in the same way and histograms of maximum resolution, respectively. See the footnote of Table 1 for the definition of the maximum resolution. Arrowheads in $(a)$ refer to characteristic signals from the chloroplast. Arrows in the histograms indicate the intensity and maximum resolution for the corresponding diffraction patterns in the left column. The total intensity represents the sum of raw diffraction intensities within the central $512 \times 512$ detector pixels on the MPCCD Octal detector, the area which does not include the missing central parts on the Dual detector. The histograms are calculated from 3135 XFEL shots for six sample support discs (a), 167 shots for two discs $(b)$ and 167 shots for three discs $(c)$. The thickness of ice was roughly estimated by monitoring with LM during sample preparation. The color scale of all the diffraction patterns is identical and shown at the top of $(a)$. 
efficient collection of optimal diffraction signals. From the sort of samples shown in Fig. 2(c), more than $65 \%$ of XFEL shots yielded diffraction signals with a resolution better than $70 \mathrm{~nm}$. A single biological object of around 1-2 $\mu \mathrm{m}$ typically diffracted X-rays to a resolution of 40-45 nm (Fig. $3 a$ ), but we sometimes observed diffraction signals beyond a resolution of $20 \mathrm{~nm}$ from biological objects probably located near the center of the beam (Takayama, Inui et al., 2015).

\subsection{Structural analysis of frozen-hydrated biological samples}

Here we describe the application of cryo-XFEL-CDI to two biological samples: chloroplasts isolated from C. melorae and minicells grown from Escherichia coli strain ME8077 (Adler et al., 1967). Complementary use of EM and LM was also quite useful in these applications, as will be demonstrated below.

We first took a correlative LM and EM approach (CLEM; de Boer et al., 2015) for the chloroplast samples. A frozenhydrated chloroplast appears green under cryo-LM, indicating the presence of chlorophylls (Fig. 4a). The same chloroplast has a nearly perfect circular shape with a dimension of $\sim 1.7 \mu \mathrm{m}$ under cryo-EM at a higher magnification (Fig. 4b). Fig. 4(c) shows a representative diffraction pattern obtained with a single XFEL shot from a frozen-hydrated chloroplast sample like that in Fig. 2(c). The statistics of the pattern are summarized in Table 1 . The speckle size of the pattern is $\sim 5 \times 5$ pixels, which is consistent with the size of a chloroplast estimated from the dimension in Fig. 4(b). As the dimension of the chloroplast is approximately three times larger than the small-angle resolution of the pattern (see \$2), many speckles near the beam center are not recorded in the pattern. Nevertheless, we succeeded in reconstructing a projected electron-density map of the chloroplast (Fig. 4d) from the diffraction pattern at an effective resolution of $192 \mathrm{~nm}$
(Table 1). This reconstruction started from a circular support with the same diameter as that observed by cryo-EM (Fig. 4b). In contrast, PR calculations failed when starting from an envelope of autocorrelation of the sample (see \$2) or a circular support with a 1.2 times larger diameter than that from the cryo-EM observation. This indicates that a reliable initial support is very important especially for a larger object. The map clearly resolves two regions with high projected electron densities. This characteristic feature is consistent with previous observations by fluorescence LM and cryo-XFEL-CDI (Takayama, Inui et al., 2015) and likely represents a stack of folded thylakoid membrane layers, which contain many photosynthetic protein complexes and chlorophylls (French, 1971). The reconstructed map is the product of the projected electron density of the sample and the amplitude of the beam with a Gaussian-like profile. This results in a gradual decrease of the density away from the beam center, but does not affect the appearance of the reconstruction seriously even when the object dimension is slightly smaller than or comparable to the beam width of $\sim 2.0 \mu \mathrm{m}$ at half-maximum amplitude.

A typical E. coli minicell has a circular cell body with a diameter of $\sim 800 \mathrm{~nm}$ and one flagellum with a diameter of $30 \mathrm{~nm}$ (Fig. 5a). Some cells lose the flagellum during growth. A single-shot diffraction pattern of a frozen-hydrated minicell shows concentric rings, reflecting the circular cell body (Fig. $5 b$ and Table 1). A projected electron-density map of the minicell (Fig. 5c) was successfully reconstructed from the diffraction pattern at an effective resolution of $52 \mathrm{~nm}$ (Fig. $5 b$ ) by using the conventional PR protocol starting from an envelope of autocorrelation. The map resolves non-uniform densities likely representing the distribution of cellular components, which were reported by EM observations with chemical fixing and thin-sectioning (Dennis \& Rogerson, 1975). Again cryoXFEL-CDI can study a whole intact cell without these treat- (a)

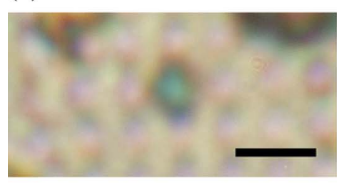

(b)

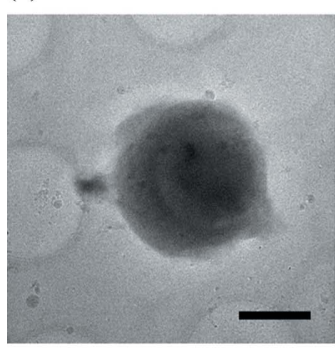

(c)

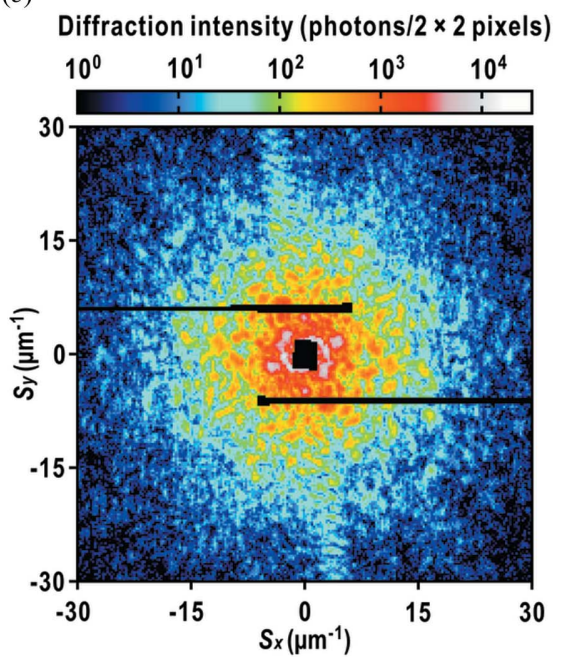

(d)

\section{Projected electron density (a.u)}

\section{0}

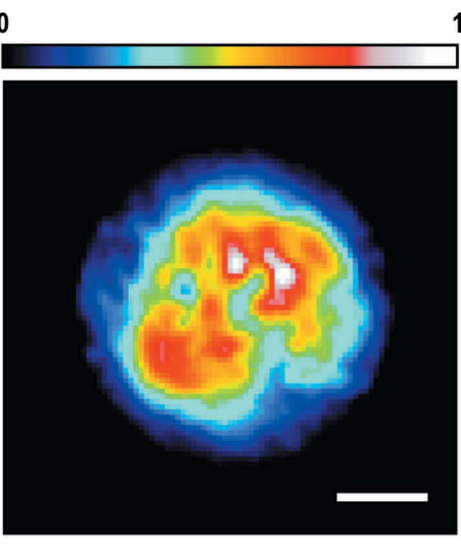

Figure 4

Structural analysis of chloroplasts isolated from $C$. merolae. Light $(a)$ and electron $(b)$ micrographs of a frozen-hydrated chloroplast isolated from $C$. merolae observed under cryogenic conditions. The sample was frozen on holey carbon film (Quantifoil Micro Tools GmbH, Germany) as in $\S 2$ and Fig. $2(a)$. Micrographs were taken at $81 \mathrm{~K}(\mathrm{LM})$ and $97 \mathrm{~K}(\mathrm{EM})$. (c) A diffraction pattern from a frozen-hydrated chloroplast. The pattern is $2 \times 2$ binned. $(d)$ A projected electron-density map of the chloroplast reconstructed from the diffraction pattern in (c). This was obtained by the HIO-SW PR, but no stable solution was obtained by the OSS PR for this pattern in $(c)$ (see $\$ 2)$. The bars represent $5 \mu \mathrm{m}(a), 1 \mu \mathrm{m}(b)$ and $500 \mathrm{~nm}(d)$. The color scales are shown at the top of the panels in $(c)$ and $(d)$. 
ments. The map also shows a flagellum-like structure sticking out from the cell body (Fig. 5c). The dimension of the single flagellum is consistent with the resolvable size from the Rayleigh criterion at this resolution. The structure is confirmed by an omit difference Fourier map (Drenth, 2007) between the observed and calculated amplitudes with the calculated phase by masking the flagellum-like density in the reconstructed map (Fig. 5d). We also found that the PR calculations led to less converged reconstructions when starting from a support excluding the flagellum-like density. To

(a)

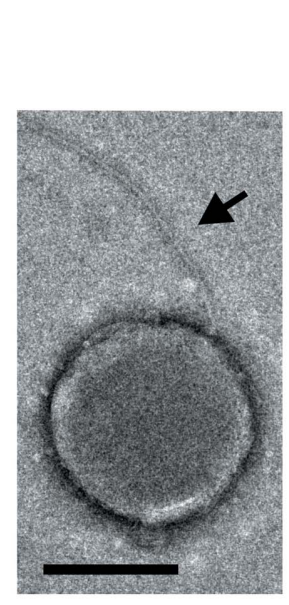

(c)

\section{Projected} 0 electron density (a.u)
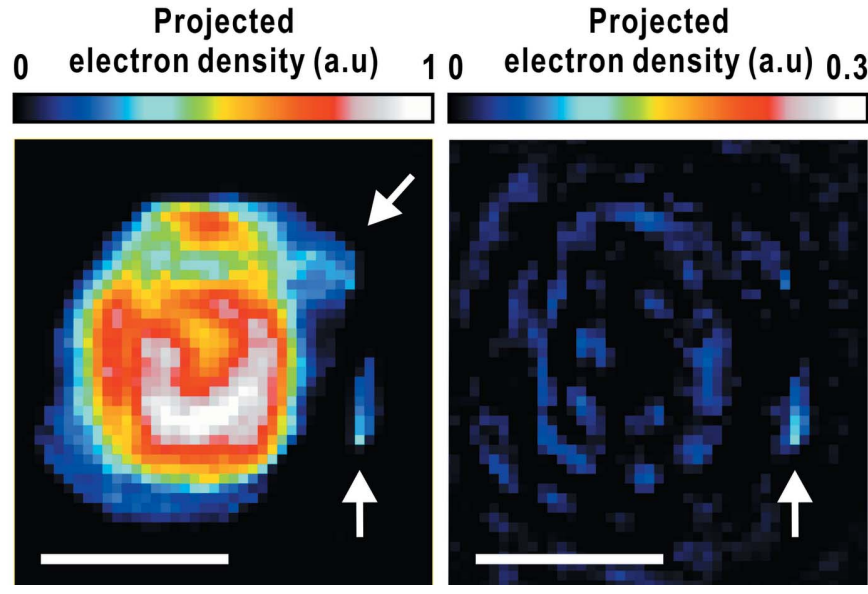

Figure 5

Structural analysis of E. coli minicells. (a) An electron micrograph of a minicell (E. coli strain ME8077) negatively stained with phosphotungstic acid. The arrow indicates a flagellum sticking out from the cell. (b) A diffraction pattern from a frozen-hydrated minicell. The pattern is $2 \times 2$ binned. (c) A projected electron-density map of the minicell reconstructed from the diffraction pattern shown in $(b)$. Arrows indicate a density similar to the flagellum. (d) An omit difference Fourier map between the observed and calculated structural amplitudes with the calculated phases by masking the flagellum-like density in the reconstructed map. The omit difference Fourier map theoretically gives the density of the omitted structure at half the weight of the original (Drenth, 2007). The bars represent $500 \mathrm{~nm}$. The color scales are shown at the top of the panels in $(b)-(d)$. confirm if the structure represents the flagellum, we need to resolve the characteristic helical structures at a much higher resolution (Yonekura et al., 2003). We are now attempting to extend the resolution by imaging with strong scatterers (see below) to resolve the flagellum and other cellular components more clearly.

\subsection{Enhancement of diffraction signals from weaker scat- terers}

As shown above, attainable spatial resolution for biological samples by current XFEL-CDI experiments has been limited to several tens of nanometres due to their poor scattering power. We proposed a method to extend the spatial resolution by imaging biological objects together with colloidal gold (CG) particles (Takayama, Maki-Yonekura et al., 2015). Weak signals from biological objects can be enhanced to a detectable level by interference with strong diffraction waves from the CGs (Shintake, 2008, 2010; Oroguchi \& Nakasako, 2013; Kim et al., 2014; Takayama, Maki-Yonekura et al., 2015). We can derive a reliable initial phase from the positions of CGs determined by the Patterson-search method as in X-ray crystallography (Jacobson \& Beckman, 1979; Sheldrick, 1997).

Here, we demonstrate the enhancement of diffraction signals from a holey silicon nitride membrane with a thickness of $100 \mathrm{~nm}$ (Norcada, Canada) by dispersing CG particles on it (Fig. 6a). Figs. 6(b) and 6(c) show a diffraction pattern with good signals and the statistics of diffraction patterns from the membrane alone. A Patterson map calculated from this diffraction pattern (Fig. $6 b$ ) reveals a ring with a diameter of $3.6 \mu \mathrm{m}$, representing a hole with a diameter of $\sim 1.8 \mu \mathrm{m}$ (Fig. $6 d$ ). Diffraction signals from the holey membrane are detected to a resolution of $26 \mathrm{~nm}$ or lower (Fig. $6 b$ and Table 1). In contrast, diffraction signals from the $\sim 80 \mathrm{~nm}$ CG particles (BBI Solutions, UK) on the holey membrane double the width of spatial frequencies (Figs. $6 e$ and $6 f$ ). The enhanced pattern (Fig. 6e) clearly resolves concentric rings characteristic of a hole on the membrane up to the edge of the diffraction pattern, proving that the resolution of signals from the hole is improved by a factor of two or more. As the direction of the enhanced signals reflects the relative position of the gold particles against the hole, the diffraction pattern (Fig. 6e) should originate from a similar field to that in Fig. $6(a)$. The interference fringes yield a pair of holographic images representing the hole on a Patterson map (Fig. $6 g$ ). The intensity of the Patterson map is amplified approximately ten times owing to the high projected electron density of the CG particles (AlsNielsen \& McMorrow, 2011).

We are now improving both the sample preparation for imaging biological targets with CG particles and the algorithm for initial phasing. As previously shown (Takayama, MakiYonekura et al., 2015), the density and arrangement of both biological targets and CG particles are critical for this approach. Again, the humidity-controlling sample preparation device and cryo-EM observation help to prepare suitable samples reproducibly (see supplementary Fig. 3 of Takayama, Maki-Yonekura et al., 2015). 
3.4. Demonstration of Patterson-search phasing for experimental diffraction patterns from distributed CG particles

When a diffraction pattern is obtained from a field containing several distributed objects as in the example for signal enhancement described above, the conventional PR calculation often fails due to a smaller OS ratio and lack of information around the center of the diffraction pattern (Miao et al., 2003; Kobayashi et al., 2014). Figs. 7(a) and 7(b) show diffraction patterns from $\sim 250 \mathrm{~nm}$ CG particles (BBI Solutions, UK). The speckle sizes of $\sim 6 \times 7$ and $\sim 8 \times 12$ pixels correspond to effective sample sizes of $\sim 1.2 \times 1.0 \mu \mathrm{m}$ and $\sim 1.2 \times 0.7 \mu \mathrm{m}$, respectively, whereas the structural information of sizes larger than $\sim 520-610 \mathrm{~nm}$, i.e. the arrangement of the CG particles, is missing due to the central beam stop. Only $15.2 \%$ and $2.4 \%$ of conventional PR reconstructions starting (a)

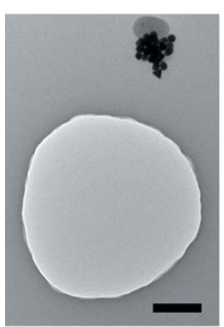

(b)

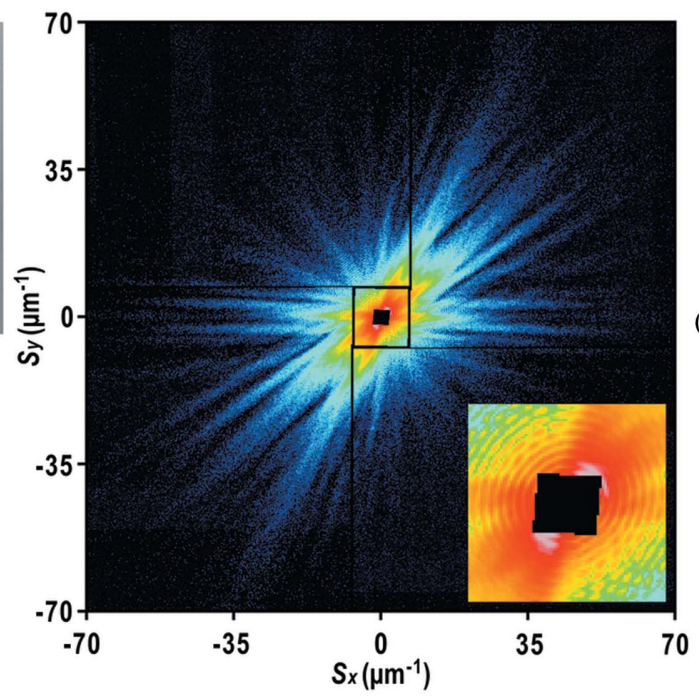

(e)

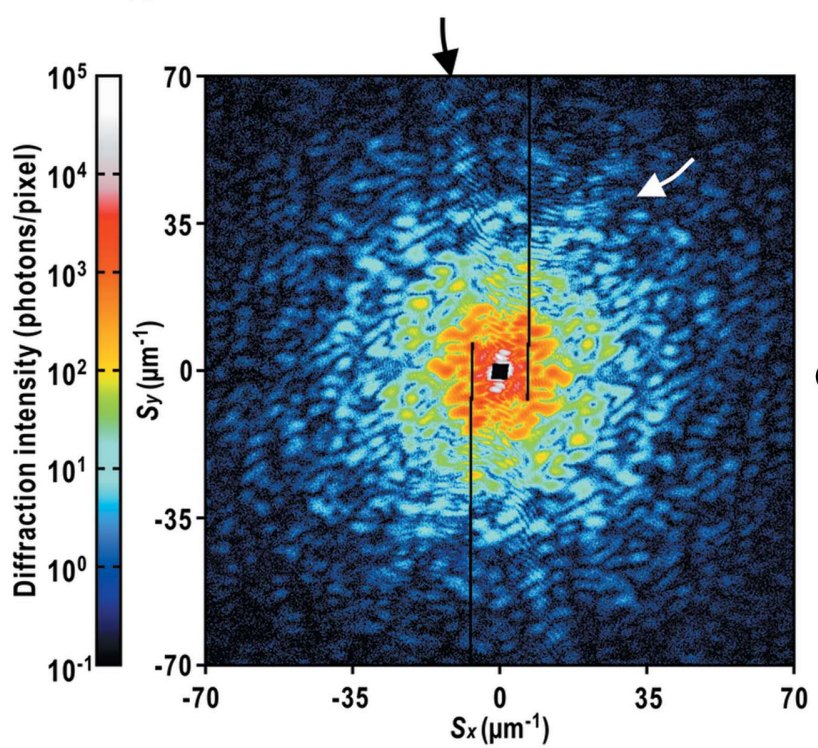

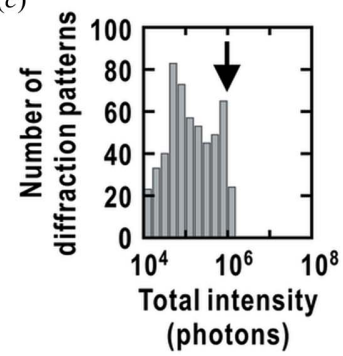

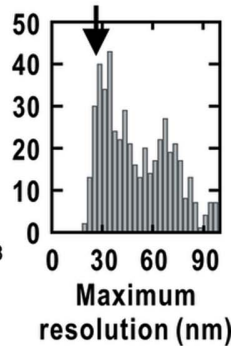

(d)

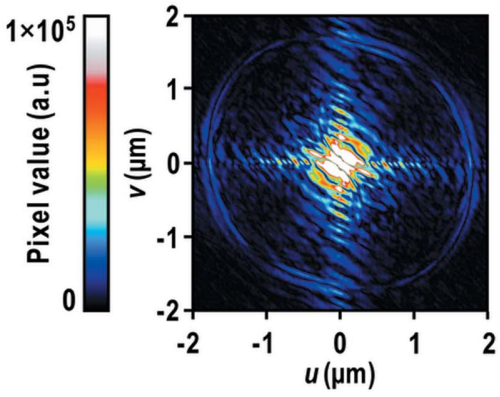

$(f)$

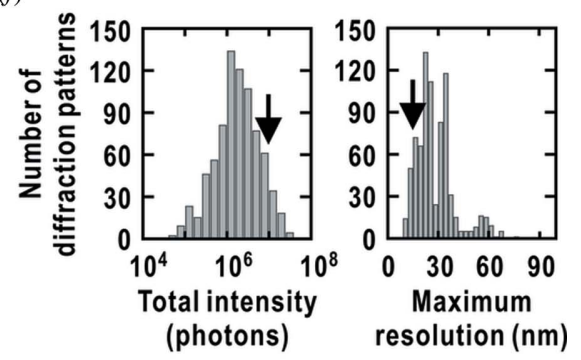

( $g$ )

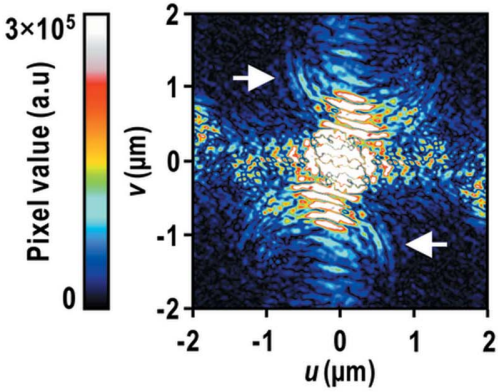

Figure 6

Enhancement of diffraction signals from holey silicon nitride membrane by interfering with signals from CG particles. (a) Electron micrograph of a cluster of CG particles on holey silicon nitride membrane. $(b),(e)$ Diffraction patterns from the holey membrane alone $(b)$ and CG particles on the holey membrane $(e)$. The inset in $(b)$ corresponds to an enlarged view of the central diffraction pattern within $\left|S_{x, y}\right| \leq 6 \mu \mathrm{m}^{-1}$ showing concentric rings characteristic of the hole. Arrows in $(e)$ indicate characteristic interference fringes extending to the edge of the pattern. $(c)$, $(f)$ Histograms of diffraction intensity and of maximum resolution of the patterns from the holey membrane alone $(c)$ and CG particles on the holey membrane $(f)$. Collected from 578 $(c)$ and 788 XFEL shots $(f)$. Arrows in the histograms in $(c)$ and $(f)$ indicate the intensity and maximum resolution for the corresponding diffraction patterns in $(b)$ and $(e)$, respectively. See also the caption of Fig. 3 for the histograms. $(d),(g)$ Patterson maps calculated from the patterns in $(b)$ and $(e)$, respectively. To minimize the effect from the missing data near the beam center, a 1 - Gaussian high-pass filter was multiplied with the diffraction patterns (Chapman et al., 2006). Autocorrelation with the hole reveals a twofold larger ring in the Patterson map (d). Cross-correlation or convolution of projected densities of the hole and the CG particles yields a pair of holographic images of the hole (arrows). The bar in $(a)$ represents $500 \mathrm{~nm}$. The color scale in $(b)$ and $(e)$ is identical and shown at the left side of $(e)$. Note that the color scale in $(g)$ is three times higher than that in $(d)$ owing to the high projected electron density of the CG particles. 
(a)

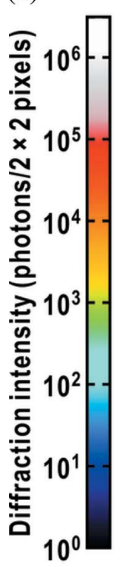

(c)

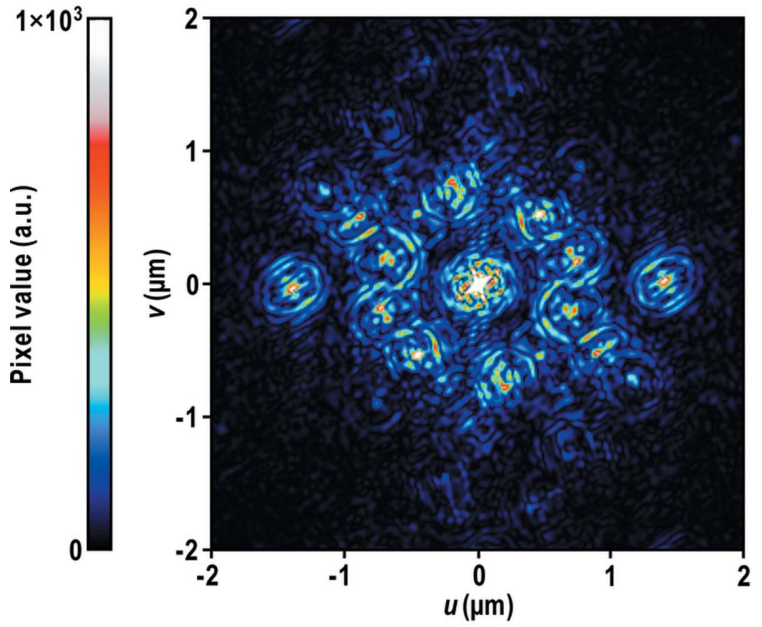

(e)
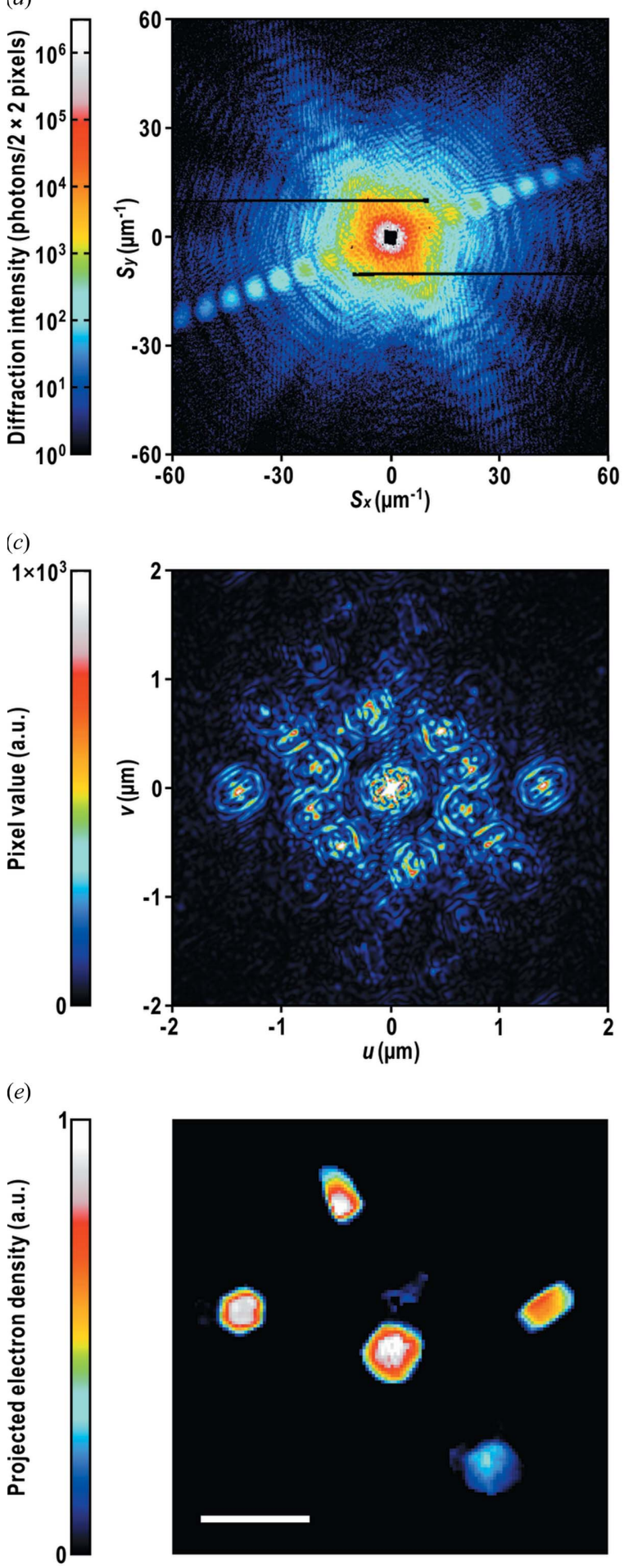

(b)

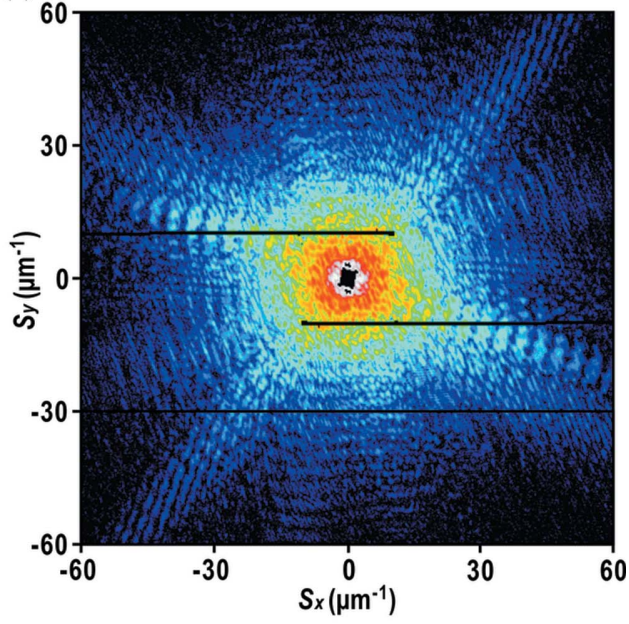

(d)

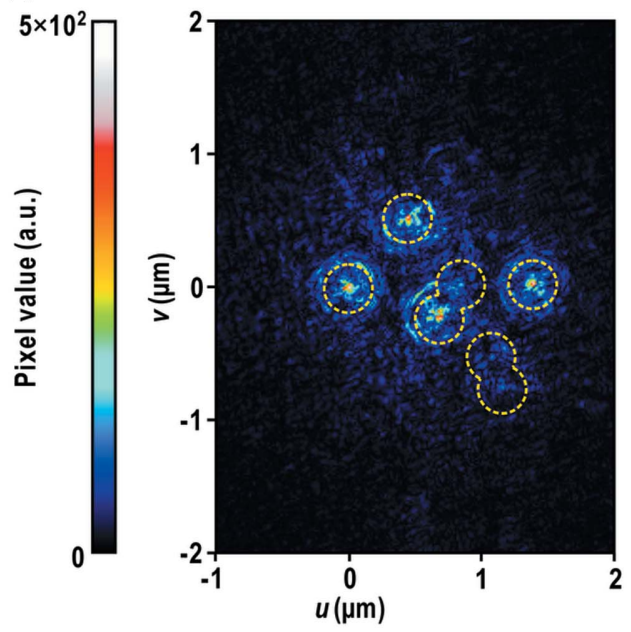

$(f)$

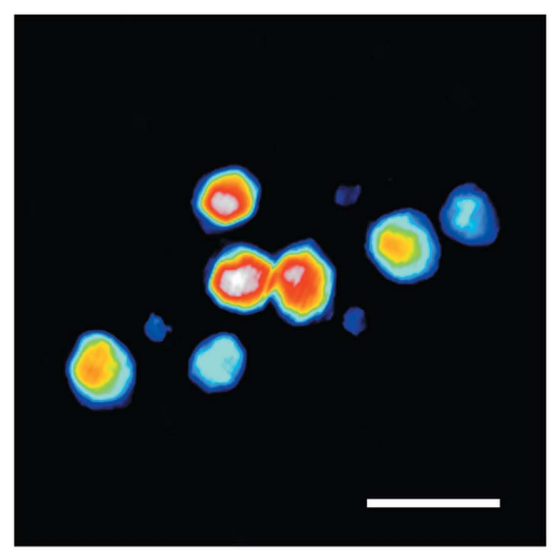

Figure 7

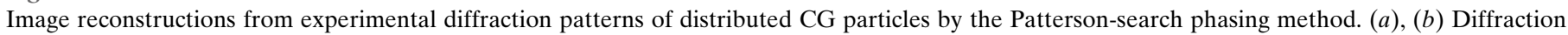

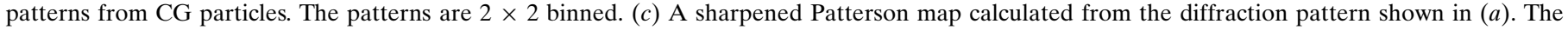

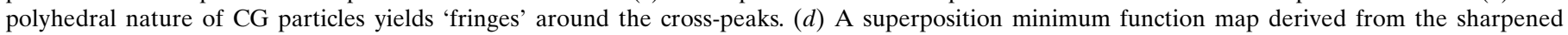

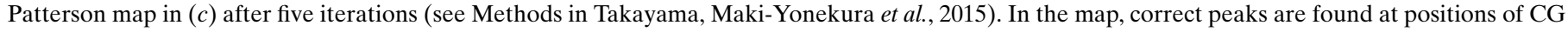

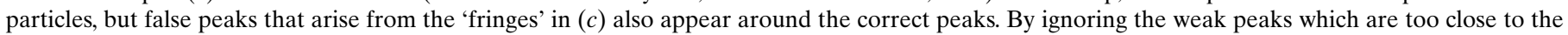

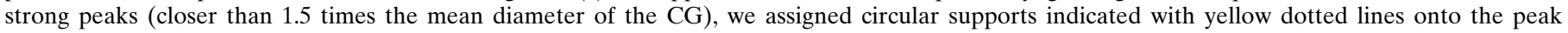

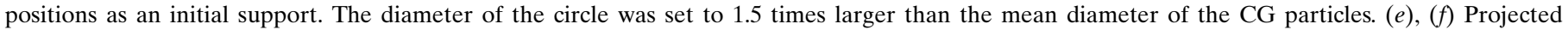

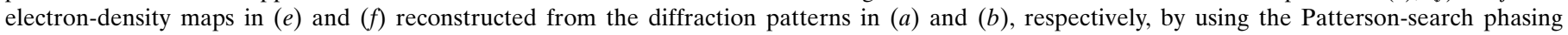

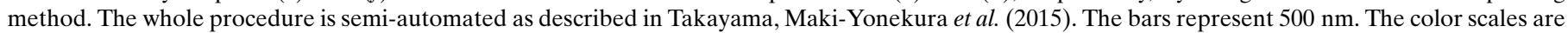
identical in the panels in the same row, and shown at the left side of each row. 
Table 2

Convergence of the HIO-SW PR by Patterson-search phasing.

The success of the reconstructions was judged based on size, shape and arrangement of the reconstructed CG particles. Failed reconstructions showed uninterpretable features or missed several CG particles during the SW process even though SW cutoff values were varied to increase the rate of PR converging to the correct solution.

\begin{tabular}{|c|c|c|}
\hline Diffraction pattern in Fig. & $7(a)$ & $7(b)$ \\
\hline No. of independent reconstructions & 500 & 165 \\
\hline \multicolumn{3}{|l|}{ No. of successful reconstructions } \\
\hline Conventional & 76 & 4 \\
\hline Patterson-search phasing & 414 & 155 \\
\hline $\begin{array}{l}R_{\mathrm{F}} \text { of successful reconstructions } \\
\text { by Patterson-search phasing }\end{array}$ & $0.295-0.347$ & $0.294-0.345$ \\
\hline Improvement by Patterson-search phasing & 5.4 times & 38.8 times \\
\hline
\end{tabular}

from an envelope of autocorrelation of the sample converged to reasonable projected electron-density maps (Table 2).

We then applied the Patterson-search phasing method (Takayama, Maki-Yonekura et al., 2015) to these patterns (Figs. $7 a$ and $7 b$ ) and successfully solved the sharpened Patterson maps (Fig. 7c). Starting from loose circular supports assigned onto the positions of the CG particles (Fig. 7d; see also the caption of Fig. 7), the number of successful reconstructions by HIO-SW calculations dramatically improved by five and 39 times (Table 2). After further refinement of the phase by using OSS (see \$2), we reconstructed projected electron-density maps of the CG particles at an effective resolution of $28.5 \mathrm{~nm}$ and $23.1 \mathrm{~nm}$ (Figs. $7 e, 7 f$ and Table 1). The convergence of the OSS calculations was also remarkable due to the reliable initial phase as previously reported (Takayama, Maki-Yonekura et al., 2015). The reconstruction clearly resolves a CG particle with a lower density in Fig. 7(e), a particle which is probably off the beam center. In contrast, conventional PR tends to remove this particle during the SW process. In addition, our protocol reconstructs well rod-like and triangular-shaped CG particles even starting from the circular supports assigned onto the CG positions. These results indicate the robustness and versatility of our method utilizing Patterson-search phasing.

\section{Conclusion}

Cryo-XFEL-CDI allows fast diffraction data collection from biological samples in a native, or closely native, state. This technique may even capture intermediate states in a cellular cycle or keep unstable cell organelles intact. Considering both the scattering power of biological samples and the convergence of the PR algorithm, objects with dimensions of 0.5$1 \mu \mathrm{m}$ are suitable for current XFEL-CDI experiments. Application to targets with dimensions of $1-2 \mu \mathrm{m}$ remains challenging but possible if a reliable initial support can be prepared using various methods such as CLEM (Fig. 4) and Patterson-search phasing (Fig. 7). Though the present attainable resolution is still not high enough to resolve macromolecular assemblies and cellular components in detail, it could be improved by using a more intense X-ray beam and/or imaging with strong scatterers. The latter approach combined with Patterson-search phasing could also solve unstable PR problems and reduce computing cost. This could be especially beneficial to XFEL-CDI, which yields a huge number of diffraction data sets.

If sample objects show good isomorphic structure, a threedimensional electron-density map can be reconstructed in the same way as cryo-EM single-particle analysis (Oroguchi \& Nakasako, 2013) or from diffraction patterns of randomly oriented objects (Ekeberg et al., 2015). For this kind of application, an irradiation area preferably contains many target objects to improve scattering signals and data collection efficiency (Fig. 7; Oroguchi \& Nakasako, 2013). The positions of these targets could also be determined by Patterson-search phasing and used as a reliable initial support.

As mentioned, efficient collection of optimal signals by cryo-XFEL-CDI needs suitable samples, e.g. a high number density of mono-dispersed objects embedded in thin ice under high humidity. A correlative approach with other imaging techniques such as cryo-EM and LM is particularly useful not only to pre-check sample distribution and ice thickness but also to determine the integrity or nature of the sample.

\section{Acknowledgements}

We thank Masayoshi Nakasako, Tomotaka Oroguchi, Yuki Sekiguchi, Amane Kobayashi, Masaki Yamamoto, Yukio Takahashi and Akihiro Suzuki for their help in data collection. We also thank Kensuke Tono, Yuichi Inubushi, Takashi Kameshima, Tetsukon Kim, Takahiro Date, Toshiyuki Murakami and the other members of the SACLA Engineering Team for their help with the alignment of the beamline optics and the diffractometer. We are grateful to Saori Maki-Yonekura for preparing CG particles on the perforated silicon nitride membrane, Sachihiro Matsunaga and Yayoi Inui for providing the chloroplast sample, and Masayoshi Nakasako and David B. McIntosh for help in improving the manuscript. The minicell-producing E. coli strain ME8077 was kindly provided by the National BioResource Project (NIG, Japan): E. coli. This work was supported by the RIKEN Special Postdoctoral Researchers Program and JSPS KAKENHI grant No. 25891033 to YT, and in part by a grant for the strategic program for $R \& D$ and the basic science interdisciplinary research project from RIKEN to KY. The XFELCDI diffraction data were collected at BL3 of SACLA (proposal Nos. 2012A8010, 2012B8037, 2013A8043, 2013B8049, 2014A8033 and 2014B8052).

\section{References}

Adler, H. I., Fisher, W. D., Cohen, A. \& Hardigree, A. A. (1967). Proc. Natl Acad. Sci. USA, 57, 321-326.

Als-Nielsen, J. \& McMorrow, D. (2011). Elements of Modern X-ray Physics, 2nd ed. Chichester: John Wiley and Sons.

Boer, P. de, Hoogenboom, J. P. \& Giepmans, B. N. G. (2015). Nat. Methods, 12, 503-513.

Chapman, H. N., Barty, A., Marchesini, S., Noy, A., Hau-Riege, S. P., Cui, C., Howells, M. R., Rosen, R., He, H., Spence, J. C. H., 
Weierstall, U., Beetz, T., Jacobsen, C. \& Shapiro, D. (2006). J. Opt. Soc. Am. A, 23, 1179-1200.

Dennis, C. A. \& Rogerson, A. C. (1975). J. Bacteriol. 124, 1610-1612.

Drenth, J. (2007). Principles of Protein X-ray Crystallography, 3rd ed. New York: Springer.

Ekeberg, T. et al. (2015). Phys. Rev. Lett. 114, 098102.

Emma, P. et al. (2010). Nat. Photon. 4, 641-647.

Fienup, J. R. (1982). Appl. Opt. 21, 2758-2769.

French, C. S. (1971). Proc. Natl Acad. Sci. USA, 68, 2893-2897.

Hantke, M. F. et al. (2014). Nat. Photon. 8, 943-949.

Hirata, K. et al. (2014). Nat. Methods, 11, 734-736.

Ishikawa, T. et al. (2012). Nat. Photon. 6, 540-544.

Jacobson, R. A. \& Beckman, D. E. (1979). Acta Cryst. A35, 339-340.

Joti, Y., Kameshima, T., Yamaga, M., Sugimoto, T., Okada, K., Abe,

T., Furukawa, Y., Ohata, T., Tanaka, R., Hatsui, T. \& Yabashi, M. (2015). J. Synchrotron Rad. 22, 571-576.

Kameshima, T., Ono, S., Kudo, T., Ozaki, K., Kirihara, Y., Kobayashi, K., Inubushi, Y., Yabashi, M., Horigome, T., Holland, A., Holland, K., Burt, D., Murao, H. \& Hatsui, T. (2014). Rev. Sci. Instrum. 85, 033110.

Kassemeyer, S. et al. (2012). Opt. Express, 20, 4149-4158.

Kim, C., Kim, Y., Song, C., Kim, S. S., Kim, S., Kang, H. C., Hwu, Y., Tsuei, K.-D., Liang, K. S. \& Noh, D. Y. (2014). Opt. Express, 22, 29161-29169.

Kimura, T., Joti, Y., Shibuya, A., Song, C., Kim, S., Tono, K., Yabashi, M., Tamakoshi, M., Moriya, T., Oshima, T., Ishikawa, T., Bessho, Y. \& Nishino, Y. (2014). Nat. Commun. 5, 3052.

Kobayashi, A., Sekiguchi, Y., Takayama, Y., Oroguchi, T. \& Nakasako, M. (2014). Opt. Express, 22, 27892-27909.

Marchesini, S., He, H., Chapman, H. N., Hau-Riege, S. P., Noy, A., Howells, M. R., Weierstall, U. \& Spence, J. C. H. (2003). Phys. Rev. $B, \mathbf{6 8}, 140101$.

McDowall, A. W., Chang, J.-J., Freeman, R., Lepault, J., Walter, C. A. \& Dubochet, J. (1983). J. Microsc. 131, 1-9.

Miao, J., Charalambous, P., Kirz, J. \& Sayre, D. (1999). Nature (London), 400, 342-344.

Miao, J., Chen, C.-C., Song, C., Nishino, Y., Kohmura, Y., Ishikawa, T., Ramunno-Johnson, D., Lee, T.-K. \& Risbud, S. H. (2006). Phys. Rev. Lett. 97, 215503.
Miao, J., Ishikawa, T., Anderson, E. H. \& Hodgson, K. O. (2003). Phys. Rev. B, 67, 174104.

Miao, J., Ishikawa, T., Robinson, I. K. \& Murnane, M. M. (2015). Science, 348, 530-535.

Nakasako, M. et al. (2013). Rev. Sci. Instrum. 84, 093705.

Neutze, R., Wouts, R., van der Spoel, D., Weckert, E. \& Hajdu, J. (2000). Nature (London), 406, 752-757.

Oroguchi, T. \& Nakasako, M. (2013). Phys. Rev. E, 87, 022712.

Oroguchi, T. et al. (2015). J. Phys. B At. Mol. Opt. Phys. 48, 184003.

Rodriguez, J. A., Xu, R., Chen, C.-C., Zou, Y. \& Miao, J. (2013). J. Appl. Cryst. 46, 312-318.

Schot, G. van der et al. (2015). Nat. Commun. 6, 5704.

Seibert, M. M. et al. (2011). Nature (London), 470, 78-81.

Sekiguchi, Y., Oroguchi, T. \& Nakasako, M. (2016). J. Synchrotron Rad. 23, 312-323.

Sekiguchi, Y., Oroguchi, T., Takayama, Y. \& Nakasako, M. (2014). J. Synchrotron Rad. 21, 600-612.

Sekiguchi, Y., Yamamoto, M., Oroguchi, T., Takayama, Y., Suzuki, S. \& Nakasako, M. (2014). J. Synchrotron Rad. 21, 1378-1383.

Sheldrick, G. M. (1997). Methods Enzymol. 276, 628-641.

Shintake, T. (2008). Phys. Rev. E, 78, 041906.

Shintake, T. (2010). Phys. Rev. E, 81, 019901.

Takahashi, Y., Suzuki, A., Zettsu, N., Oroguchi, T., Takayama, Y., Sekiguchi, Y., Kobayashi, A., Yamamoto, M. \& Nakasako, M. (2013). Nano Lett. 13, 6028-6032.

Takayama, Y., Inui, Y., Sekiguchi, Y., Kobayashi, A., Oroguchi, T., Yamamoto, M., Matsunaga, S. \& Nakasako, M. (2015). Plant Cell Physiol. 56, 1272-1286.

Takayama, Y., Maki-Yonekura, S., Oroguchi, T., Nakasako, M. \& Yonekura, K. (2015). Sci. Rep. 5, 8074.

Takayama, Y. \& Nakasako, M. (2012). Rev. Sci. Instrum. 83, 054301.

Tono, K., Togashi, T., Inubushi, Y., Sato, T., Katayama, T., Ogawa, K., Ohashi, H., Kimura, H., Takahashi, S., Takeshita, K., Tomizawa, H., Goto, S., Ishikawa, T. \& Yabashi, M. (2013). New J. Phys. 15, 083035 .

Yonekura, K., Maki-Yonekura, S. \& Namba, K. (2003). Nature (London), 424, 623-650.

Yumoto, H. et al. (2012). Nat. Photon. 7, 43-47. 